\title{
A INCORPORAÇÃO DA RÉGUA DE CÁLCULO NO ENSINO DE MULTIPLICAÇÃO ATRAVÉS DA SUA CONSTRUÇÃO E DO SEU MANUSEIO
}

\section{THE INCORPORATION OF SLIDE RULES IN MULTIPLICATION EDUCATION THROUGH ITS CONSTRUCTION AND HANDLING}

\author{
Andressa Gomes dos Santos ${ }^{1}$ \\ Instituto Federal de Educação, Ciências e Tecnologia do Ceará \\ Ana Carolina Costa Pereira ${ }^{2}$ \\ Universidade Estadual do Ceará
}

\begin{abstract}
Resumo
A história da matemática vem sendo discutida em pesquisas de vertente historiográfica atualizada, como uma fornecedora de meios potencialmente didáticos para o ensino de Matemática. Dentre os diversos recursos que ela pode oferecer, podemos citar os instrumentos matemáticos, especialmente, os elaborados nos séculos XVI e XVII. Dessa forma, a Régua de Cálculo Linear, desenvolvida por William Oughtred (1574 - 1660), é uma opção de recurso histórico que pode ser utilizado no ensino de Matemática. Esse instrumento auxilia em operações aritméticas como multiplicação e divisão, usando princípios dos logaritmos e pode ser estudada e moldada para sua incorporação no ensino de Matemática. Nesta pesquisa, trataremos, especificamente, do ensino de multiplicação por meio desse instrumento. Este artigo é um fragmento de um trabalho de conclusão de curso de licenciatura em Matemática, da Universidade Estadual do Ceará, do ano de 2019. O artigo apresenta um pouco da ideia dos logaritmos de Napier, a construção da Régua de Cálculo Linear e como manuseá-la, trazendo aspectos históricos dos logaritmos, estudos de conceitos matemáticos dos séculos XVI e XVII, que podem ser percebidos na Régua de Cálculo e algumas contribuições que a construção e manuseio desse instrumento podem acarretar na construção do conhecimento matemático. A pesquisa tem como objetivo conhecer as potencialidades didáticas da construção e manipulação da Régua de Cálculo Linear para o ensino de multiplicação. Portanto, a pesquisa é qualitativa, de cunho bibliográfico e aborda a construção dos logaritmos de John Napier (1550 - 1617), da Régua de Cálculo e suas contribuições para o ensino de Matemática.
\end{abstract}

Palavras-chave: Ensino de multiplicação; Régua de Cálculo Linear; História da matemática.

1 andressagomes009@gmail.com

2 carolina.pereira@uece.br

Número Especial - IV Seminário Cearense de História da Matemática

Boletim Cearense de Educação e História da Matemática - Volume 07, Número 20, 357 - 369 (2020)

DOI: $10.30938 /$ bocehm.v7i20.2827 
A incorporação da régua de cálculo no ensino de multiplicação através da sua construção e do seu manuseio

Andressa Gomes dos Santos e Ana Carolina Costa Pereira

\begin{abstract}
The history of mathematics has been discussed in updated historiographic research as a provider of potentially didactic means for teaching mathematics. Among the many resources it can offer we can cite the mathematical instruments, especially those elaborated in the 16th and 17th centuries. Thus, the Linear Calculus Ruler developed by William Oughtred $(1574-1660)$ is a historical resource option that can be used in mathematics teaching. This instrument assists in arithmetic operations such as multiplication and division using logarithmic principles and can be studied and shaped for incorporation into mathematics teaching. In this research we will deal specifically with the teaching of multiplication through this instrument. This article is a fragment of a work that concludes the Mathematics Degree Course of the State University of Ceará in 2019. The article presents a little of the idea of Napier's logarithms, the construction of the Linear Calculus Ruler and how to handle it. , bringing historical aspects of the logarithms, studies of mathematical concepts of the sixteenth and seventeenth century that can be perceived in the Calculation Rule and some contributions that the construction and handling of this instrument can entail in the construction of mathematical knowledge. The research aims to know the didactic potentialities of the construction and manipulation of the Linear Calculation Ruler for the teaching of multiplication. Therefore, the research is qualitative in bibliographic nature and addresses the construction of the logarithms of John Napier (1550 - 1617), the Ruler of Calculus and their contributions to the teaching of mathematics.
\end{abstract}

Keywords: Multiplication teaching; Linear slide rule; Mathematics history.

\title{
Introdução
}

O ensino de Matemática está em pauta em vários estudos com a intenção de apresentar novos métodos de construir conhecimento matemático. Com isso, a história da matemática se encaixa como uma opção de encontrar meios para ensinar um conceito matemático.

No âmbito das pesquisas com a temática voltada para o ensino de matemática com o uso de recursos históricos, a historiografia atualizada se faz presente pela maneira de apresentar a história da matemática, pois essa tendência historiográfica não enfatiza os conceitos matemáticos e seus estudiosos, mas foca no “[...] processo da construção desses mesmos conteúdos, métodos, técnicas ou algoritmos historicamente contextualizados" (SAITO, 2018, p. 8).

Assim, a historiografia atualizada permite que a história da matemática seja descrita salientando os conceitos matemáticos e seus desenvolvimentos de forma historicamente contextualizada. 
A incorporação da régua de cálculo no ensino de multiplicação através da sua construção e do seu manuseio

Andressa Gomes dos Santos e Ana Carolina Costa Pereira

Dessa maneira, a história pode oferecer alguns recursos potencialmente didáticos para o ensino. Dentro das possibilidades de meios didáticos, podemos destacar os instrumentos históricos que tiveram maior destaque nos séculos XVI e XVII.

Nesse período, diversos instrumentos matemáticos foram desenvolvidos, dentre instrumentos para medição de altura e distâncias, astrolábios e esferas armilar, também foram construídos instrumentos para facilitar operações aritméticas, como a Régua de Cálculo (SAITO; DIAS, 2011).

A Régua de Cálculo foi desenvolvida por William Oughtred e, nela, estão contidos conceitos de logaritmos para simplificar cálculos matemáticos, como multiplicação, divisão, encontrar logaritmo decimal, realizar operacões com expoente e extração de raízes quadradas e cúbicas, além de apresentar escalas trogonométricas (MENDES; SOARES, 2019).

Neste artigo, tratamos apenas da noção por trás da construção dos logaritmos desenvolvidos por Napier e estudos posteriores sobre esses logaritmos, para adentrarmos na construção da Régua de Cálculo e das escalas logarítmicas para realizar operações de multiplicação e divisão, porém abordaremos somente como manipular as escalas para obter resultados para multiplicações.

Para isso, utizamos uma pesquisa bibliográfica que, conforme Prodanov e Freitas (2013), trata-se de uma pesquisa baseada em fontes secundárias, como livros, artigos, teses e dissertações. Fizemos um apanhado de fontes para construirmos as escalas logarítmicas, para o embasamento histórico e para o estudo do desenvolvimento e construção dos logaritmos de Napier.

Logo, a pesquisa é qualitativa, que é “[...] uma categoria de investigação que tem como objeto o estudo de uma unidade de forma aprofundada, podendo tratar-se de um sujeito, de um grupo de pessoas, de uma comunidade etc.” (PRODANOV; FREITAS, 2013, p. 60), pois tratamos dos resultados da construção da Régua de Cálculo que possam ser inseridos no ensino de Matemática, especialmente, no ensino de multiplicação.

Dessa maneira, o presente artigo tem como objetivo conhecer as potencialidades didáticas da construção e manipulação da Régua de Cálculo Linear para o ensino de multiplicação. 
A incorporação da régua de cálculo no ensino de multiplicação através da sua construção e do seu manuseio

Andressa Gomes dos Santos e Ana Carolina Costa Pereira

\title{
Ideias iniciais dos logaritmos segundo Napier
}

John Napier é um dos principais nomes associados ao desenvolvimento dos logaritmos, além dele, podemos citar Henry Briggs (1561 - 1631), que reformulou os logaritmos de Napier para os que conhecemos atualmente, os logaritmos na base 10.

Sobre os logaritmos, Napier publicou, em 1614, uma obra em que os apresenta, Mirifici Logarithmorum Canonis Descriptio. Nessa publicação, o autor não adicionou detalhes sobre os métodos usados na construção dos logaritmos, somente a descrição deles (HOBSON, 1914).

O método é apresentado em uma publicação de seu filho, Robert Napier, em 1619, após a morte de seu pai. Mirifici Logarithmorum Canonis Constructio traz o processo de construção dos logaritmos de Napier, que se constituíram baseados na ideia de progressões.

Segundo Pereira (2015, p. 24):

\begin{abstract}
O procedimento adotado por Napier para a criação dos logaritmos tem como base as relações de Stifel, que consistia em um método que envolvia duas relações matemática: as Progressões Aritméticas e Geométricas. Ele observou que o produto ou o quociente de dois termos quaisquer de uma Progressões Geométrica estava associado à soma ou diferença dos termos de uma Progressão Aritmética.
\end{abstract}

Na obra Constructio, é apresentado como foi feita a construção dos logaritmos, que ocorreu por meio do que conhecemos, hoje, por Progressão Geométrica (PG) e Progressão Aritmética (PA). Na relação entre PG e PA, construída por Napier, foi possível simplificar cálculos de multiplicação e divisão para soma e subtração por meio dos logaritmos.

Antes de descrevermos o método de construção dos logaritmos de Napier, precisamos entender algumas nomenclaturas e notações da época, como o raio e as casas decimais, que foram se modificando com o passar do tempo e se padronizando a partir do século XIX, para o que conhecemos hoje.

Na obra Constructio, Napier explica o porquê de ter utilizado $10^{7}$ como raio. Napier (1619, p. 6, tradução nossa) diz:

Quanto a 100000, que o mais rudimentar tomado para o valor máximo do seno, o mais instruído é adotar 10000000, do qual todos os senos podem ser expressos com melhor discriminação. Assim, estamos usando o mesmo número para o seno máximo e para o máximo das Proporções Geométricas. 
A incorporação da régua de cálculo no ensino de multiplicação através da sua construção e do seu manuseio

Andressa Gomes dos Santos e Ana Carolina Costa Pereira

Para Napier, era mais conveniente usar o raio de $10^{7}$ unidades e esse também era o valor máximo que o seno poderia assumir. Dessa maneira, ele obteria maior precisão nos cálculos com as progressões.

Outra notação utilizada era o uso de ponto para separar casas decimais. Na "nossa notação atual para números com o ponto decimal parece ter sido independentemente inventada por Napier [...]" (HOBSON, 1914, p. 22, tradução nossa). Napier utilizava um ponto para identificar a parte decimal do número. Dito isso, podemos descrever o método no qual Napier desenvolveu os logaritmos.

\section{Construção dos logaritmos de Napier}

Visto um pouco das obras de Napier, que descreveram e apresentaram a construção dos logaritmos e depois de definirmos alguns termos acerca das notações usadas para sua construção, discorreremos como se deu a construção desse conceito matemático.

Para Napier, de acordo com Pereira (2015), a construção dos logaritmos inicia propondo duas retas, uma marcada com Progressões Aritméticas crescentes e outra, com Progressões Geométricas decrescentes. Na Figura 1, podemos observar a semirreta $\overrightarrow{\mathrm{PT}}$, dividida em segmentos de reta $\left(\overline{\mathrm{PP}_{1}}, \overline{\mathrm{P}_{1} \mathrm{P}_{2}}, \overline{\mathrm{P}_{2} \mathrm{P}_{3}}, \overline{\mathrm{P}_{3} \mathrm{P}_{4}}, \overline{\mathrm{P}_{4} \mathrm{P}_{5}}\right)$, sendo $\mathrm{P}$ o ponto inicial representado por 0 e $\mathrm{T}$ um ponto genérico na semirreta. Essa semirreta está enumerada com uma Progressão Aritmética, em que y representa um número natural qualquer.

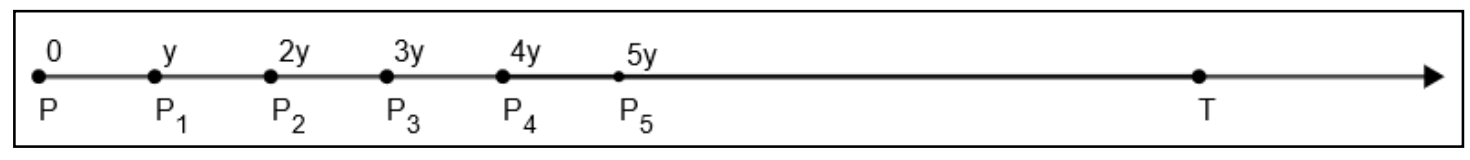

Figura 1 - Semirreta da Progressão Aritmética.

Fonte: Elaborada pelas autoras.

Na Progressão Aritmética, o segmento de reta $\overline{\mathrm{PP}_{1}}=\overline{\mathrm{P}_{1} \mathrm{P}_{2}}=\overline{\mathrm{P}_{2} \mathrm{P}_{3}}=\overline{\mathrm{P}_{3} \mathrm{P}_{4}}=\overline{\mathrm{P}_{4} \mathrm{P}_{5}}=\ldots$ $=\mathrm{y}$, pois considerando, por exemplo, $\mathrm{y}=1, \overline{\mathrm{PP}_{1}}=\overline{\mathrm{P}_{1} \mathrm{P}_{2}}=\overline{\mathrm{P}_{2} \mathrm{P}_{3}}=\overline{\mathrm{P}_{3} \mathrm{P}_{4}}=\ldots=1$, a razão da PA é 1. Podemos generalizar, considerando y um número natural qualquer, que será a razão da PA. Além disso, percebemos que os segmentos, na semirreta $\overrightarrow{\mathrm{PT}}$, têm o mesmo comprimento.

Já na Figura 2, a semirreta $\overrightarrow{\mathrm{SV}}$ está marcada com a Progressão Geométrica, em que r é o raio $\left(10^{7}\right)$ e também é o maior valor das Progressões Geométricas e x é um 
A incorporação da régua de cálculo no ensino de multiplicação através da sua construção e do seu manuseio

Andressa Gomes dos Santos e Ana Carolina Costa Pereira

número próximo a 1, então, Napier considerou 0,9999999. Assim, cada ponto $\left(S, S_{1}, S_{2}\right.$, $\left.\mathrm{S}_{3}, \mathrm{~S}_{4}\right)$ é a distância dele mesmo até o ponto $\mathrm{V}$.

\begin{tabular}{|llllll|}
0 & $\mathrm{rx}$ & $\mathrm{rx}^{2}$ & $\mathrm{rx}^{3}$ & $\mathrm{rx}^{4}$ & \\
\hdashline & $\mathrm{S}_{1}$ & $\mathrm{~S}_{2}$ & $\mathrm{~S}_{3}$ & $\mathrm{~S}_{4}$ & $\mathrm{~V}$ \\
\hline
\end{tabular}

Figura 2 - Semirreta da Progressão Geométrica.

Fonte: Elaborada pelas autoras.

$\mathrm{O}$ segmento $\overline{\mathrm{SS}_{1}}=\overline{\mathrm{SV}}-\overline{\mathrm{S}_{1} \mathrm{~V}}=\mathrm{r}-\mathrm{rx} . \mathrm{O}$ segmento $\overline{\mathrm{SS}_{1}}$ equivale ao segmento $\overline{\mathrm{SV}}$ menos o segmento $\overline{\mathrm{S}_{1} \mathrm{~V}}$, que é o mesmo que considerarmos r-rx (lembrando que $\mathrm{r}=10^{7}=\overline{\mathrm{SV}}$, que é a maior das Progressões Geométricas).

Podemos deduzir, a partir disso, que:

$$
\begin{aligned}
& \overline{\mathrm{SS}_{2}}=\overline{\mathrm{SV}}-\overline{\mathrm{S}_{2} \mathrm{~V}}=\mathrm{r}-\mathrm{rx}^{2} \\
& \overline{\mathrm{SS}_{3}}=\overline{\mathrm{SV}}-\overline{\mathrm{S}_{3} \mathrm{~V}}=\mathrm{r}-\mathrm{rx}^{3} \\
& \overline{\mathrm{SS}_{4}}=\overline{\mathrm{SV}}-\overline{\mathrm{S}_{4} \mathrm{~V}}=\mathrm{r}-\mathrm{rx}^{4}
\end{aligned}
$$

Sabendo que cada segmento é igual a $\mathrm{r}$ menos sua respectiva Progressão, temos, colocando r e rx em evidência:

$$
\begin{gathered}
\overline{\mathrm{SS}_{1}}=\mathrm{r}-\mathrm{rx}=\mathrm{r}(1-\mathrm{x}) \\
\overline{\mathrm{SS}_{2}}=\mathrm{r}-\mathrm{rx}^{2}=\mathrm{rx}(1-\mathrm{x}) \\
\overline{\mathrm{SS}_{3}}=\mathrm{r}-\mathrm{rx}^{3}=\mathrm{rx}^{2}(1-\mathrm{x}) \\
\overline{\mathrm{SS}_{4}}=\mathrm{r}-\mathrm{rx}^{4}=\mathrm{rx}^{3}(1-\mathrm{x})
\end{gathered}
$$

Segundo Katz (2010 apud PEREIRA, 2015), considerando um ponto L e um ponto Q, respectivamente, na semirreta, com as marcações das Progressões Aritméticas (ver Figura 3) e na reta das Progressões Geométricas, com tais pontos se movimentando sobre as progressões com velocidade constante e os dois pontos partindo do zero, quando $\mathrm{L}$ atingir a e Q um ponto c, tal que a distância, a partir de S, é b, dizemos que a é o logaritmo de $b$. 
A incorporação da régua de cálculo no ensino de multiplicação através da sua construção e do seu manuseio

Andressa Gomes dos Santos e Ana Carolina Costa Pereira

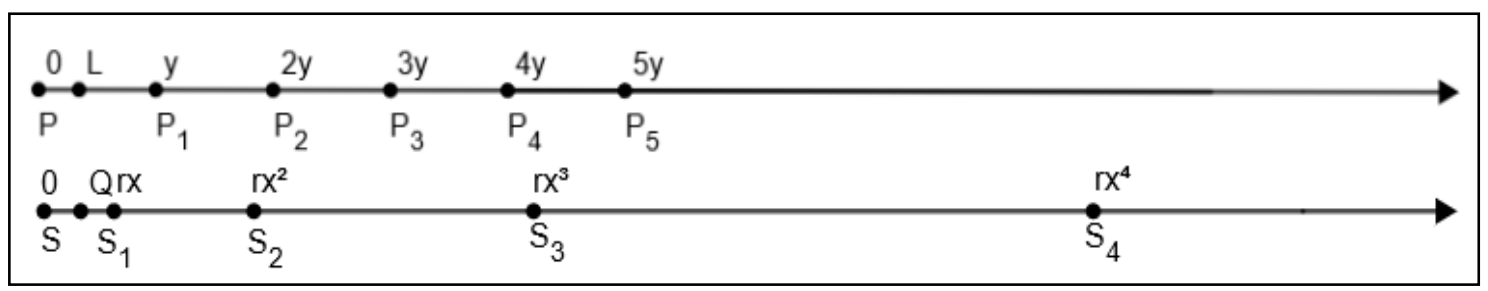

Figura 3 - Semirretas da PA e PG marcadas com os pontos L e Q.

Fonte: Elaborada pelas autoras.

Com L e Q se movimentando em suas respectivas semirretas com velocidades iguais, L se movimentando aritmeticamente e Q se deslocando geometricamente, temos que, quando L alcançar um a qualquer e Q alcançar um c qualquer, tal que a distância de $\mathrm{S}$ até c é b, Napier denominou que a é o logaritmo de b (Napier representou, algebricamente, como Naplog $b=a)$.

Desse modo, o Naplog do segmento $\overline{\mathrm{SS}_{1}}$ é $\overline{\mathrm{PP}_{1}}$, mas já vimos que $\overline{\mathrm{SS}_{1}}=\mathrm{r}(1-\mathrm{x})$ $\mathrm{e} \overline{\mathrm{PP}_{1}}=0 \mathrm{y}$, de outra forma:

$$
\text { Naplog } \overline{\mathrm{SS}_{1}}=\overline{\mathrm{PP}_{1}} \text { ou Naplog } \mathrm{r}(1-\mathrm{x})=0 \mathrm{y}
$$

Partindo dessa ideia, Napier construiu uma tabela de senos, utilizando o raio de $10^{7}$ e usou o número mais próximo de 1 com 7 casas decimais para o valor de $\mathrm{x}$, assim, utilizou 0,9999999. Substituindo esses valores em Naplog $r(1-x)=0 y$, temos:

$$
\begin{gathered}
\text { Naplog }\left[10^{7}(1-0,9999999)\right]=0 \\
\text { Naplog }\left[10^{7}(0,0000001)\right]=0 \\
\text { Naplog }[1]=0
\end{gathered}
$$

Napier observou que: $0,9999999=1-\frac{1}{10^{7}}$, pois $1-\frac{1}{10^{7}}=\frac{10^{7}-1}{10^{7}}=$ $\frac{10000000-1}{10^{7}}=\frac{9999999}{10^{7}}=\frac{9999999}{10000000}=0,9999999$. "Então, ele calculou as potências de 0,9999999, de $\mathrm{x}^{2}$ até $\mathrm{x}^{50}$ subtrações sucessivas, que consistiu na sua primeira tabela de logaritmos [...]" (PEREIRA, 2015, p. 27). Na Figura 11, podemos ver a primeira tabela que Napier construiu, partindo desse princípio das sucessivas subtrações. 


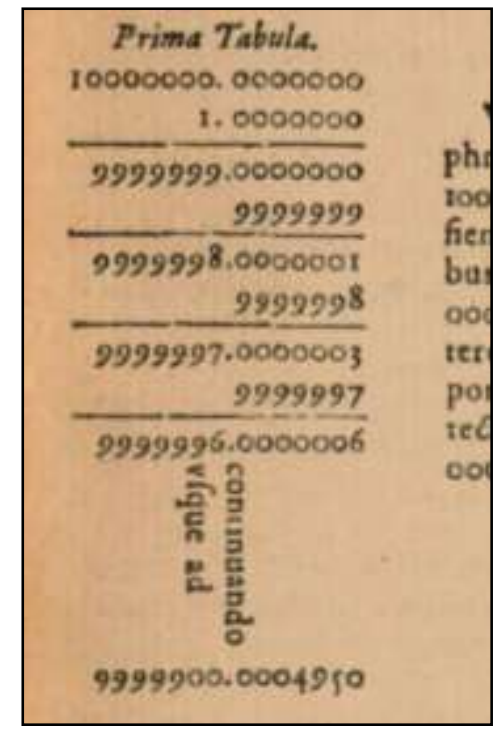

Figura 4 - Prima Tabula.

Fonte: Napier (1619, p. 9).

Na primeira tabela dos logaritmos, Napier utilizou o ponto para separar as casas decimais. Na tabela da Figura 4, Napier faz quatro subtrações partindo da potência de $10^{14}$ subtraindo $10^{7}$, em seguida, subtraindo 0,9999999 do resultado. E ele continuou até a subtração resultar no número 9999900,0004950.

Com a construção dos logaritmos e a percepção de que as operações de divisão e de multiplicação poderiam ser simplificadas com esse método, alguns estudiosos utilizaram esse conceito para desenvolver instrumentos matemáticos que pudessem ajudar em cálculos.

\section{A Régua de Cálculo Linear: escalas para operação de multiplicação}

A Régua de Cálculo Linear foi construída a partir dos logaritmos de Napier, dos logaritmos de Henry Briggs na base 10 e começando da construção das escalas logarítmicas de Gunter. Com elas, foi possível transformar multiplicações em somas, divisões em subtração e é esse o princípio da Régua de Cálculo: facilitar cálculos por meio da soma ou subtração de logaritmos. Construímos as escalas logarítmicas da multiplicação, conforme Gunter fez em suas escalas e explicou seu manuseio.

Para construção das escalas logarítmicas, utilizaremos as definições de logaritmo na base $10\left(\log _{10}\right.$ ou, simplesmente, $\left.\log \right)$, que Henry Briggs adotou a partir dos logaritmos de Napier. No Quadro 1, podemos ver os resultados dos logaritmos de 1 a 10. 
A incorporação da régua de cálculo no ensino de multiplicação através da sua construção e do seu manuseio

Andressa Gomes dos Santos e Ana Carolina Costa Pereira

Quadro 1 - Logaritmos na base.

\begin{tabular}{|c|c|}
\hline Logaritmo & Valor \\
\hline $\log 1$ & 0 \\
\hline $\log 2$ & 0,301029995 \\
\hline $\log 3$ & 0,477121254 \\
\hline $\log 4$ & 0,602059991 \\
\hline $\log 5$ & 0,698970004 \\
\hline $\log 6$ & 0,77815125 \\
\hline $\log 7$ & 0,84509804 \\
\hline $\log 8$ & 0,903089987 \\
\hline $\log 9$ & 0,954242509 \\
\hline Log 10 & 1 \\
\hline \multicolumn{2}{|c|}{ Fonte: Elaborado pelas autoras. } \\
\hline
\end{tabular}

Dessa maneira, construímos as escalas logarítmicas (Figura 5), de acordo com Gunter e da notação de logaritmos na base 10 .

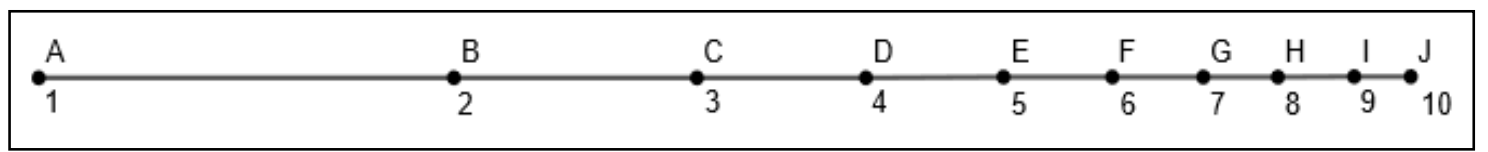

Figura 5 - Escala logarítmica segundo Gunter.

Fonte: Elaborada pelas autoras.

Assim, o segmento $\overline{\mathrm{AB}}=\log 2=0.301029995$, da mesma forma, para o segmento $\overline{\mathrm{AC}}=\log 3=0,477121254$. Então, cada segmento, partindo do ponto $\mathrm{A}=1$ do segmento de reta $\overline{\mathrm{AJ}}$, equivale a um respectivo logaritmo. Em outras palavras: $\overline{\mathrm{AC}}=\overline{\mathrm{AB}}+\overline{\mathrm{BC}}=\log 2+(\log 3-\log 2)=0,301029995+0,176091259=0,48^{3}$ (ver representação Figura 6).

\begin{tabular}{|ccccc|}
\hline$A$ & & $B$ & $C$ \\
1 & 0.3 & 2 & 0.18 & 3 \\
\hline
\end{tabular}

Figura 6 - Princípio de construção da escala de Gunter.

Fonte: Elaborada pelas autoras.

A partir disso, "as operações de multiplicações e divisões, na escala, eram efetuadas pela soma e subtração de segmentos (segmentos nos quais estão representando logaritmos), ou seja, soma e subtração de logaritmos" (PEREIRA, 2015, p. 52).

De acordo com Pereira (2015), os segmentos representam os logaritmos. Logo, o segmento $\overline{\mathrm{AB}}=\log 2, \overline{\mathrm{AC}}=\log 3, \overline{\mathrm{AD}}=\log 4, \overline{\mathrm{AE}}=\log 5, \overline{\mathrm{AF}}=\log 6, \overline{\mathrm{AG}}=\log 7$, etc.

\footnotetext{
${ }^{3}$ Esse é o resultado da aproximação do valor de $\log 2$ e $\log 3$.
} 
A incorporação da régua de cálculo no ensino de multiplicação através da sua construção e do seu manuseio

Andressa Gomes dos Santos e Ana Carolina Costa Pereira

Porém, de acordo com Martins, Pereira e Fonseca (2016), se utilizarmos a Régua no tamanho real da escala de Gunter, ela seria muito pequena para a manipulação. Então, usamos uma escala de ampliação, na qual a distância gráfica (d) é maior que a distância real (D).

Como vimos, $\log 10=1=\overline{\mathrm{AJ}}=\mathrm{D}$, D sendo o tamanho real da escala. $\mathrm{E}$ representamos a Régua $10=$ d. Dessa maneira, nossa Régua será 10 vezes maior que a régua original. Assim,

$$
\begin{gathered}
10(\log 2)=10(0,3)=3=\overline{\mathrm{AB}} \\
10(\log 3)=10(0,48)=4,8=\overline{\mathrm{AC}} \\
10(\log 4)=10(0,6)=6=\overline{\mathrm{AD}} ; \\
\cdots \\
10(\log 10)=10(1)=10=\overline{\mathrm{AJ}} .
\end{gathered}
$$

Como as multiplicações e divisões são transformadas em somas e subtrações, se quisermos saber qual o resultado da multiplicação de 2 por 5 , basta considerarmos o segmento $\overline{\mathrm{AB}}+\overline{\mathrm{AE}}=\log 2+\log 5=\log (2 \times 5)=\log 10=\overline{\mathrm{AJ}}^{4}$

De fato, os segmentos representam os logaritmos e as operações de multiplicação se transformam em soma, para a escala de Gunter, ele precisava de um compasso para realizar as operações. Oughtred inseriu outra escala para obter de imediato os resultados das operações realizadas.

\section{Manuseio da régua de multiplicação}

De acordo com Martins, Pereira e Fonseca (2016), para realizarmos o produto na Régua de Cálculo Linear, devemos, primeiramente, alinhar o multiplicando, localizado na segunda régua, com o número 1 da primeira régua e, posteriormente, localizar, na primeira régua, o multiplicador desejado. Localizado o multiplicador, temos abaixo dele, na segunda régua, o nosso produto.

Então, na Figura 7, temos a Régua de multiplicação, as escalas logarítmicas são as mesmas da escala de Gunter. Na Régua de Cálculo linear, contamos com duas escalas logarítmicas, uma fixa A e outra deslizante B.

\footnotetext{
${ }^{4}$ Pela propriedade de $\log$ aritmo, $\log a+\log b=\log (a \times b)$.
} 
A incorporação da régua de cálculo no ensino de multiplicação através da sua construção e do seu manuseio

Andressa Gomes dos Santos e Ana Carolina Costa Pereira

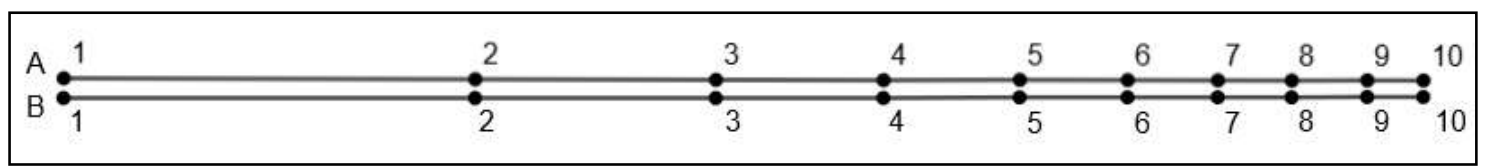

Figura 7 - Régua de multiplicação.

Fonte: Elaborada pelas autoras.

Na Figura 8, temos a Régua A fixa e a Régua B que desliza, na multiplicação, a Régua B irá deslizar para esquerda e posicionar no 1 da Régua A o número que deseja ser multiplicado. Por exemplo, se desejarmos multiplicar 2 por 5, posicionaremos o 2 , localizado na Régua B, no 1 da Régua A e localizaremos, na mesma Régua, o número 5 e, abaixo dele, na Régua B, estará o resultado da multiplicação de 2 por 5 (veja figura 8).

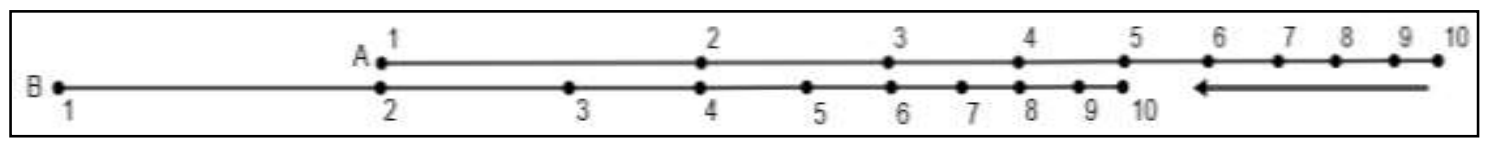

Figura 8 - Duas escalas logarítmicas deslizantes.

Fonte: Elaborada pelas autoras.

Na régua de multiplicação de Oughtred, utilizam-se duas escalas logarítmicas, sendo uma deslizante para obter resultados imediatos. Por exemplo, na Figura 8, podemos ver que o ponto $2(\log 2)$, na segunda régua, está posicionada no ponto 1 da primeira régua. $\mathrm{E}$ temos os resultados, assim, dos números multiplicados por 2, até o 5.

Podemos construir tantas marcações quanto quisermos para obter os resultados desejados das operações que, eventualmente, podem ser feitas. Pois, se quisermos os resultados da multiplicação por 10, deveríamos construir uma régua com mais marcações além de somente 10 . Ou, intuitivamente, multiplicar a segunda régua por 10, assim, ao invés da régua começar por 1 , iria começar com o marcador 10.

Com isso, cálculos de multiplicação, utilizando a régua de cálculo linear, ficam mais simples por meio da soma de logaritmos. Podemos, desse modo, obter o resultado de qualquer número desejado, basta posicioná-lo corretamente na Régua.

Uma opção de uso, em sala de aula, é a construção da Régua de Cálculo somente com os números primos. Como exemplo, temos a Figura 9, marcando com os números primos de 2 a $10^{5}$, o professor pode estipular outro intervalo para construção.

\footnotetext{
${ }^{5} \mathrm{O}$ número 1 não é primo, mas será marcado na escala por questão de facilitar o manuseio para obter os demais resultados compostos. O professor pode levar isso em consideração e reforçar que o número 1 não é primo.
} 
A incorporação da régua de cálculo no ensino de multiplicação através da sua construção e do seu manuseio

Andressa Gomes dos Santos e Ana Carolina Costa Pereira

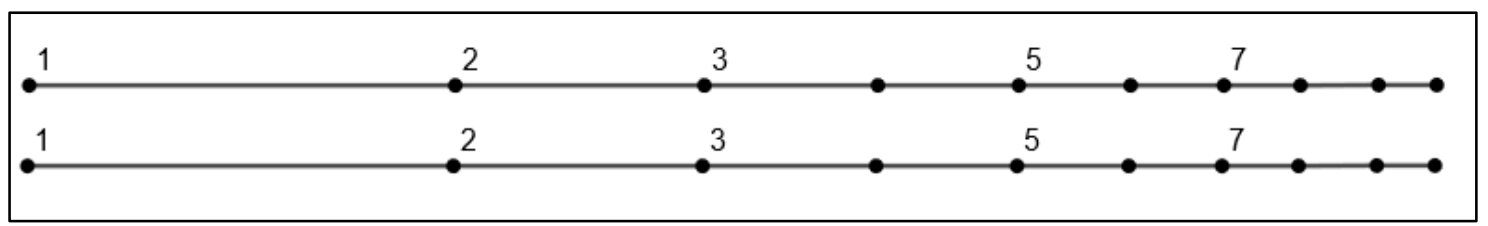

Figura 9 - Escala logarítmica dos números primos.

Fonte: Elaborada pelas autoras.

Com as escalas logarítmicas marcadas com os números primos, os alunos podem manusear a régua de multiplicação e obter os demais números compostos da régua. Escolhendo o número 3 para multiplicação, por exemplo, obtemos a seguinte escala representada na Figura 10. Os resultados estão representados com a cor vermelha.

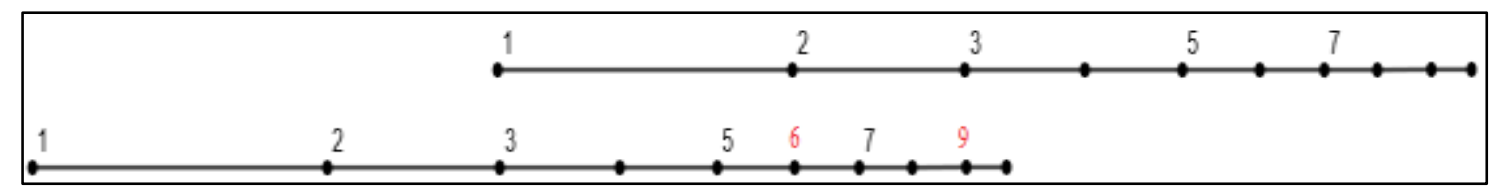

Figura 10 - Multiplicação do algarismo 3.

Fonte: Elaborada pelas autoras.

Logo, o professor pode estimular a multiplicação por meio da construção das escalas logarítmicas da Régua de Cálculo e reforçar e construir conceitos matemáticos como os de números primos e compostos, além da multiplicação propriamente dita.

\section{Considerações finais}

O artigo trouxe reflexões acerca da história da matemática no ensino de Matemática e uma maneira inovadora pautada em uma vertente historiográfica atualizada, para que o professor possa utilizá-la em sala de aula e, dessa forma, construir conhecimentos ou ressignificá-los.

A história pode fornecer vários recursos para efetivar seu uso no ensino, um dos meios didáticos podem ser os instrumentos históricos que incorporem algum conceito matemático, que possam ser explorados.

A Régua de Cálculo é uma opção de instrumento histórico que pode ser inserido no ensino de Matemática, especificamente, no ensino de multiplicação através da sua construção e do seu manuseio.

Com este artigo, podemos conhecer um pouco da provável história por trás dos logaritmos de Napier e seu desenvolvimento, como ele foi importante na época para a construção de instrumentos de cálculo, como a Régua de Cálculo Linear de Oughtred. E, 
A incorporação da régua de cálculo no ensino de multiplicação através da sua construção e do seu manuseio

Andressa Gomes dos Santos e Ana Carolina Costa Pereira

com a construção e o manuseio desse instrumento, podemos perceber maneiras de usá-lo no ensino de multiplicação e para definir alguns conceitos matemáticos.

\section{Referências}

HOBSON, E.W. John Napier and the Invention of the logaritms, 1614, Cambridge: University Press. 48 p. 1914. Disponível em:

<http://archive.org/details/rabdologiae00napi>. Acesso em: 19 fev de 2019.

MARTINS, Eugeniano Brito; PEREIRA, Ana Carolina Costa; FONSECA, Paulo Henrique Sousa. Redescobrindo o conceito de logaritmo por meio da construção da régua de cálculo linear. Revista Eletrônica Debates em Educação Científica e Tecnológica, v. 3, p. 47-65, 2016.

MENDES, Iran Abreu; SOARES, Evanildo Costa. LogAritmos(números da razão): enfoques históricos, epistemológicos e escolares. São Paulo: Livraria da Física, 2019. (Série história da matemática para professores).

NAPIER, J. Mirifici Logarithmorum Canonis Constructio. Edinburgh: Andrew Hart, 1619.

PEREIRA, Ana Carolina Costa. Aspectos Históricos da régua de cálculo para a construção de conceitos matemáticos. São Paulo: Livraria da Física, 2015. (História da Matemática para o Ensino).

PRODANOV, C. C.; FREITAS, E. C. Metodologia do trabalho científico: Métodos e Técnicas da Pesquisa e do Trabalho Acadêmico. 2. ed. Novo Hamburgo: Editora Feevale, 2013.

SAITO, Fumikazu. CONSTRUINDO INTERFACES ENTRE HISTÓRIA E ENSINO DA MATEMÁTICA. Ensino de Matemática em Debate, São Paulo, p.3-19, 2016. Disponível em: <https://revistas.pucsp.br/index.php/emd/article/view/29002>. Acesso em: 15 ago. 2018.

SAITO, Fumikazu; DIAS, Marisa da Silva. Articulação de entes matemáticos na construção e utilização de instrumentos de medida do século XVI. In: GONÇALVES, Carlos Henrique Barbosa; ALVES, Eva Maria Siqueira (Org.). Coleção história da matemática para professores. Aracaju: IX Seminário Nacional de História da Matemática, 2011. p. 7-63.

Articulação de entes matemáticos na construção e utilização de instrumentos de medida do século XVI. In: GONÇALVES, Carlos Henrique Barbosa; ALVES, Eva Maria Siqueira (Org.). Coleção história da matemática para professores. Aracaju: Ix Seminário Nacional de História da Matemática, 2011. p. 7-63. 